REVIEWS

\title{
Systematic review of predictors of infant care competence among women with postpartum depression
}

\author{
Debbie Jones $^{1}$, Nicole Letourneau ${ }^{2,3}$, Linda Duffett-Leger*4 \\ ${ }^{1}$ Medical Sciences Graduate Program, Cumming School of Medicine, University of Calgary, Calgary, Alberta, Canada \\ ${ }^{2}$ Owerko Centre at the Alberta Children's Hospital Research Institute, University of Calgary, Calgary, Alberta, Canada \\ ${ }^{3}$ Faculty of Nursing, University of Calgary, Alberta, Canada \\ ${ }^{4}$ Faculty of Nursing and Cumming School of Medicine, Departments of Pediatrics, Psychiatry, \& Community Health Sciences, \\ University of Calgary, Calgary, AB, Canada
}

Received: November 20, 2018

DOI: $10.5430 /$ jnep.v9n5p118
Accepted: January 16, 2019

Online Published: January 29, 2019

\begin{abstract}
Postpartum depression (PPD) is a serious illness that affects mothers worldwide. The symptoms of PPD such as low mood and fatigue undermine the quality of mothers' interactions with their children, likely explaining the less than optimal development of children of mothers with PPD. In this way, PPD reduces mothers' Infant Care Competence (ICC), that is, mothers' perceived and performed infant caregiving ability. ICC is similar to other concepts such as maternal competence; however, ICC is specific in its focus on a mother's perceptions of her infant care abilities. Knowledge of predictors of ICC in the context of PPD would inform interventions for mothers with PPD to increase maternal caregiving quality, preventing negative long-term effects on children's development. Thus, an integrative systematic review was completed to determine predictors of ICC (both performed and perceived abilities) in the context of PPD. Six electronic databases were searched (MEDLINE, PubMed, PsycINFO, Cumulative Index to Nursing and Allied Health Literature [CINAHL], SocINDEX, and the Cochrane Library) for relevant studies that met search criteria. Twenty-one eligible articles were obtained. Results revealed variables that explained ICC in the context of maternal PPD including: depression severity and timing of depressive symptoms, social support, maternal adversities, infant characteristics, as well as demographic variables such as education and income. Overall, this review provides insight into common explanatory variables of ICC that could be used to target interventions in the postpartum period to promote maternal caregiving abilities, and ultimately children's development and health in the context of PPD.
\end{abstract}

Key Words: Postpartum depression, Infant care competence, Maternal-infant interaction, Predictors

\section{INTRODUCTION}

Postpartum depression (PPD) is a mental health disorder that affects approximately $15 \%$ of women in Canada $^{[1]}$ and up to $15 \%$ of women worldwide. ${ }^{[2]}$ Depression with peripartum onset may be diagnosed during pregnancy or within four weeks after delivery ${ }^{[3]}$ and symptoms include decreased anhedonia, feelings of helplessness and hopelessness, and chronic low mood. ${ }^{[3]}$ However, many studies refer to depression occurring in the 1st postpartum year as PPD. ${ }^{[4]} \mathrm{PPD}$ negatively impacts Infant Care Competence (ICC), defined as

\footnotetext{
*Correspondence: Linda Duffett-Leger; Email: linda.duffettleger@ucalgary.ca; Address: Faculty of Nursing, University of Calgary, 2500 University Drive NW, Calgary, AB, Canada.
} 
a mother's perceived ability and performance in infant care tasks. ${ }^{[5]}$ ICC is similar to other concepts such as maternal competence ${ }^{[6]}$ or role attainment, ${ }^{[7]}$ but is more specific to the infant care role. While maternal role competence refers to how well a mother carries out her role and maternal role attainment is the process in which a mother accepts her role, both involve aspects of ICC, that is, mothers' perceptions of ability and/or performance of infant care tasks. Other related terms include maternal self-efficacy, ${ }^{[8]}$ maternal selfesteem, ${ }^{[9]}$ maternal-infant interaction including sensitivity and responsiveness, ${ }^{[10,11]}$ and maternal-child relationship quality, ${ }^{[12]}$ all of which include aspects of ICC, including performance and perceptions of infant care abilities. In this way, ICC may be considered a more omnibus term in relation to infant care. Understanding predictors of ICC, especially among depressed mothers whose caregiving quality are at risk due to the symptoms of depression, would offer guidance for nurses working with these families to promote maternal and infant health.

PPD is well known to interfere with performed ICC, especially maternal-infant interaction quality, as the symptoms of PPD often render mothers less sensitive and attentive towards infant cues. ${ }^{[13]}$ Such reduced ICC can be damaging to infant and child development, ${ }^{[14]}$ including long-term emotional and cognitive deficits as well as health problems. ${ }^{[1,15]}$ Moreover, even when mothers' depressive symptoms resolve, the quality of interactions with children remain disrupted, ${ }^{[14]}$ which likely overlaps with how mothers perceive or perform ICC. ${ }^{[16]}$ Identifying variables that explain ICC in these mothers may be useful to understand and target intervention.

Systematic reviews have examined associations between PPD and infant/child cognitive and behavioural development, ${ }^{[17]}$ maternal-infant interaction ${ }^{[11]}$ and the benefits of treatment of PPD ${ }^{[18]}$ on maternal-infant interaction and child development, including psychosocial development; ${ }^{[15]}$ however, reviews have not focused on ICC or mothers' perceptions of abilities to care for infants and how these were affected by PPD. To our knowledge, neither primary studies nor reviews have examined predictors of ICC and related concepts among mothers affected by PPD. Outside of the context of PPD, studies have examined predictors of ICCrelated concepts such as parenting self-efficacy and maternal role competence. ${ }^{[6,19,20]}$ Identified predictors include social support, child development, severity of depressive symptoms, preterm birth, child temperament, and medications. ${ }^{[19]}$ Social support may reduce depressive symptoms, ${ }^{[21]}$ but the degree to which social support may affect ICC of women with PPD is not known. Researchers have reported improved symptoms when a woman receives adequate support from a partner or spouse but this support has not demonstrated an

Published by Sciedu Press impact on the maternal-child interaction quality. ${ }^{[22]}$ It is important to study whether quality of social support influences ICC for mothers with depression.

Another predictor of ICC in the context of PPD may include severity of depressive symptoms. More severe symptoms of depression including mental confusion and even paranoid thoughts, delusions or bizarre behaviors, ${ }^{[23]}$ that may or may not be co-morbid with postpartum psychosis, ${ }^{[24]}$ can affect ICC and especially maternal-infant relationships. ${ }^{[25]}$ Further, while medications such as anti-depressants or hormone therapy for PPD have demonstrated PPD symptom relief, they do not seem to offer any improvement in the quality of maternal-child interactions, and potentially by extension, ICC. ${ }^{[15,26]}$

Further, there are no studies, however, that reveal how child development may affect or explain perceived ICC in women with PPD. An association has been observed between maternal depression and ICC, specifically, maternal-infant interaction in families with preterm infants, specifically with mothers being less attuned to infants' needs during interactions. ${ }^{[27]}$ Preterm infants may also have physical conditions and have difficulty with emotional self-regulation thus affecting ICC. Mothers of these preterm infants sometimes demonstrate more controlling behaviors or are less sensitive with their infants, ${ }^{[27]}$ which may relate to ICC. Further research is needed to determine if a child's development influences ICC among mothers with PPD. Similarly, child temperament, either difficult or compatible ${ }^{[28]}$ may relate to ICC, especially in the context of PPD. Depressed mothers often perceive their infants as more difficult ${ }^{[29,30]}$ which could undermine their ICC and relatedly, maternal-child interaction quality or maternal competence. On the other hand, a child that provides unclear cues or who is more difficult to soothe, as in the case of a colicky infant, is known to undermine maternal-infant interaction quality, ${ }^{[31]}$ and likely ICC. However, in the context of PPD, the degree to which infant temperament may influence ICC is not well understood.

While we have summarized the sparse literature on predictors of depressed mothers' abilities to care for their infants, there may be many more variables that impact ICC. Given the presumed importance of ICC to children's development in families affected by PPD, identifying predictors of ICC would be useful to identify and target interventions. As such, an integrative systematic review ${ }^{[29]}$ was conducted to determine predictors of ICC among mothers with PPD. The research question addressed is: "How do depression and other variables influence and/or explain ICC in the context of PPD?" 


\section{MeTHODS}

The steps outlined by Whittemore, Knafl ${ }^{[29]}$ including problem identification, literature search, data evaluation, data analysis and synthesis were followed in this integrative review. ICC expert Loretta Secco ${ }^{[5]}$ was also closely consulted throughout the process.

\subsection{Study selection}

A systematic search was completed using six electronic databases: MEDLINE, PubMed, PsycINFO, Cumulative Index to Nursing and Allied Health Literature (CINAHL), SocINDEX, and the Cochrane Library. There were no restrictions with regards to time period. Search terms used throughout all databases were postpartum depression OR postnatal depression OR post-partum depression OR postnatal depression. These terms were also combined with infant care competence, as well as related concepts including maternal sensitivity, maternal responsiveness, maternal role attainment, maternal-infant interaction and maternal-infant relationships. Additionally, the search was performed using the term "parenting" or "parent" substituted for the term mothering or maternal.

Studies were selected if they met the following criteria: measure or describe ICC (which includes both performed and perceived variables/concepts), using tools, questionnaires or themes that measure ICC; refer to ICC as an outcome; participants included depressed mothers identified via physician diagnosis or a validated depression screening tool at $<24$ months postpartum; were found in English-published journals; were peer reviewed; were primary studies; and were qualitative or quantitative studies. More specifically, with respect to ICC, papers were included if they measured mothers' perceived ability and performance of infant caregiving ability or observed mothers' performance of infant caregiving ability. Papers were excluded if they did not provide data to answer the research question. Abstracts were reviewed that met inclusion criteria independently. For any abstracts for which it was unclear if the study met criteria, the full article was retrieved for full assessment.

\subsection{Data extraction}

Data were extracted using a form developed specifically for the study that included author(s), study year, study design, study methods, participant description (e.g. age of mothers and infants), sample size, how ICC was measured and the results of the study were assessed. The main outcome of this review was ICC and similar concepts with different names. Dr. Loretta Secco determined similar concepts to ICC with different names, for the purpose of this narrative systematic review.

\subsection{Data summarizing and analytic strategies}

Once data were extracted, as per Whittemore, Knafl ${ }^{[29]}$ recommendations, analysis included noting patterns and themes, clustering, counting, contrasting and comparing, noting relationship between variables and identifying intervening factors. Further, the quality of the studies was assessed using an adaptation of Young, Solomon's ${ }^{[32]} 10$ criteria. The first author scored each paper, then a second author checked the scores for accuracy and agreement. Scores were derived from the assessment questions until an overall score was established. Some example questions include: 'What type of research question is being asked?' and 'Did the study methods address the most important potential sources of bias?' According to Young, \& Solomon, ${ }^{[32]}$ different study designs present with different sources of bias such as longitudinal or cohort studies. For example, there were concerns with how sample populations were selected or the length of time the samples were studied. Researchers should address how selection criteria were accounted for. If researchers addressed the studies as described according to the criteria, a point was provided. Studies were assigned a point if criteria were met or a score of zero if criteria were not met or if the study was unclear. Scores were then added up to yield a total score out of 10. The studies were then categorized as: high quality (scores 9 or 10), good (scores 6 to 8), fair (scores 4 or 5), and poor (scores 3 or less). The first author appraised the eligible studies. The majority of studies $(n=19)$ were deemed as good with scores ranging from 7 to 8 .

Three articles were considered as high quality receiving scores of 9 .

\section{RESULTS}

The systematic search identified a total of 994 articles once duplicates were removed. All abstracts were screened for eligibility and a total of 954 articles were excluded. Forty full texts were appraised for suitability and 19 articles were removed that did not meet criteria for postpartum depression and ICC. Thus, the final review includes 21 papers that met inclusion criteria. The selection process is summarized in Figure 1.

Out of the 21 eligible studies, nineteen papers were quantitative studies and two articles were qualitative. ${ }^{[33,34]}$ The ages of participants ranged from 18 to 45 years for mothers and the infants' ages ranged from 3 months to 14 months. The majority of the papers were completed in Europe (England, Finland, Sweden, Norway, Germany, Scotland; $n=8)^{[35-42]}$ and eight studies were completed in the United States. ${ }^{[43-50]}$ The sample further consisted of two papers from South America (Brazil); ${ }^{[13,34]}$ two studies from Australia; ${ }^{[33,51]}$ and one paper from Asia (Singapore). ${ }^{[20]}$ The sample of mothers 
ranged in ethnicity, marital status, and parity.

Qualitative studies. Using a hermeneutic approach, Barr ${ }^{[33]}$ studied how PPD impacts maternal adaptation by interviewing and reviewing participant's reflective journals. Researchers used units of meaning (UOM) to look for patterns and then interviews were conducted to analyze data. Frizzo, Vivian, Piccinini, Lopes ${ }^{[34]}$ explored forms of parent communication used by depressed mothers. Several interviews were completed which were recorded and transcribed. Data were then examined using an iterative approach revealing themes.

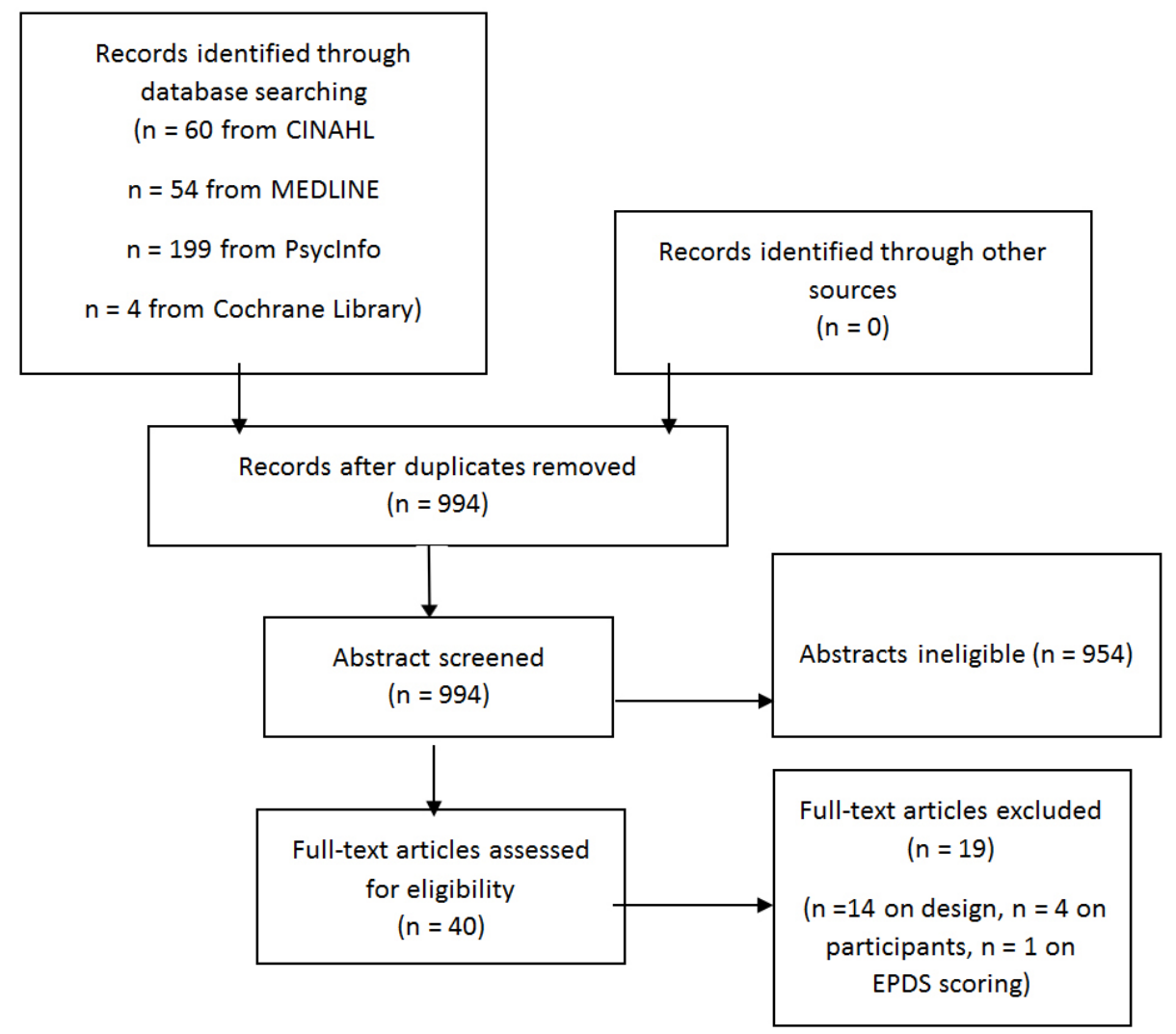

Figure 1. The selection process of articles

Quantitative studies. A variety of study designs and scales were used to describe how PPD relates to ICC. Studies involved tests of interventions including longitudinal designs ( $\mathrm{n}$ $=9),{ }^{[11,35,37-42,44]}$ randomized controlled trials $(\mathrm{n}=2),{ }^{[43,45]}$ observational $(\mathrm{n}=1),{ }^{[48]}$ correlational study designs $(\mathrm{n}=$ $2),{ }^{[13,50]}$ cohort studies $(\mathrm{n}=1),{ }^{[51]}$ non-randomized longitudinal descriptive approach $(\mathrm{n}=1),{ }^{[36]}$ and cross-sectional and longitudinal studies $(\mathrm{n}=1) .{ }^{[49]}$

Among the longitudinal study designs, measurement of ICC included videos to observe performed ICC $(n=2) .{ }^{[13,42,44]}$ Three studies used a combination of video observations as well as questionnaires to assess perceptions of ICC. ${ }^{[35,38,43]}$ Other studies $(n=4)^{[36,39,49]}$ administered questionnaires to the mothers and/or conducted interviews. One study observed the mother's interactions, which was then followed by an interview. ${ }^{[41]}$ Similarly in the randomized controlled trial conducted by Forman, O'Hara, Stuart, Gorman, Larsen, Coy, ${ }^{[45]}$ interactions were videotaped and then subsequently

Published by Sciedu Press coded.

Among all of the observational studies, ICC was measured by video in the home or in a lab with times varying from 5 minutes to 30 minutes $(n=3) .{ }^{[37,47,48]}$ In the research using correlational designs and the non-randomized longitudinal descriptive study, mothers were given questionnaires. ${ }^{[20,46,50]}$ Using questionnaires, Foreman, Henshaw ${ }^{[51]}$ studied the mother's perceptions of their infants. Completed questionnaires by participants were analyzed by using least squares scaling. Finally, the eligible qualitative studies included questionnaires, along with semi-structured and in depth interviews. ${ }^{[33,34]}$

\subsection{Depression measures}

To assess PPD or symptoms of depression in the postpartum period, nine different scales were used. The most commonly used depression scale used was the Edinburgh Postnatal Depression Scale (EPDS). Eleven studies assessed for depressive symptoms using the EPDS, however using five differ- 
ent cutoff criteria. Four studies had a cutoff score of 9 or greater. ${ }^{[37,39,40,51]}$ Two had cutoffs of greater than $10 . .^{[38,49]}$ One other study used the criteria of 12 or greater ${ }^{[50]}$ Scores between 12 and 13 were also utilized in two studies. ${ }^{[2,46]}$ One other study implemented a cutoff of greater than or equal to $13 .^{[20]}$ In the study by Murray, Friori-Cowley, Hooper, Cooper, ${ }^{[41]}$ it was unclear what criteria were applied, however interviews were completed using the Standardized Psychiatric Interview (SPI).

Only one study used the Inventory to Diagnose Depression (IDD). ${ }^{[45]}$ This is the only paper that used this 22 -item scale that is commonly used to diagnose Major Depression Disorder according to the DSM-III. ${ }^{[52]}$ In this study, those who had met the criteria for depression were then interviewed using the Structured Clinical Interview (SCID). ${ }^{[45]}$ An additional scale identified was the Symptom Checklist-90 (SCL-90) revised version. ${ }^{[48]}$ The SCL-90 is a self-report scale that includes eight fields with a focus on psychiatric symptoms. There are additional subsections within the scale with a focus on depressive symptoms. ${ }^{[53]}$ One study used the Schedule for Affective Disorders and Schizophrenia (SADS) and the Center for Epidemiological Studies Depression Scale (CES-D $)^{[44]}$ while another study used the CES-D to assess for depression. ${ }^{[35]}$ Three studies used the Beck Depression Inventory (BDI) ${ }^{[13,34,43]}$ and one used the BDI and the Depression Adjective Check List (DACL) combined to screen for depression. ${ }^{[47]}$

\subsection{Infant care competence}

The majority of the studies $(\mathrm{n}=10)$ examined performed ICC from the perspective of maternal-infant interaction quality or parent-child interaction. ${ }^{[34-37,41-44,47,49]}$ Studies that assessed perceived ICC measured maternal-infant bonding $(\mathrm{n}=2)^{[40,50]}$ and maternal sensitivity $(\mathrm{n}=2) .{ }^{[13,38]}$ Maternal feelings ${ }^{[51]}$ and perceptions of the maternal-infant relationship were further examined with respect to perceived ICC. ${ }^{[39]}$ In one study by Kim, Teti, Cole ${ }^{[48]}$ maternal emotional availability was examined. Other forms of ICC included maternal responsiveness, ${ }^{[45]}$ maternal-parental self-efficacy, ${ }^{[20]}$ maternal role attainment ${ }^{[46]}$ and maternal adaptation. ${ }^{[33]}$

\subsection{Quantitative measures and qualitative characteriza- tion of infant care competence}

Performed and perceived ICC were measured in various ways. Nearly half of the papers studied maternal-infant interactions by videotape and then coded by an outside observer. These observations were either completed in the lab or in the home of the mothers. One particular study by Campbell, Cohn, Meyers ${ }^{[44]}$ observed and videotaped interactions of mothers and their infants at 2, 4, and 6 months. Additional research assessed interactions at 6,12 , and 18 months. ${ }^{[35]}$ Other studies used questionnaires or interviews to observe interaction. One study used videotape observations as well as an interview. ${ }^{[35]}$ To study maternal-infant interaction, mothers were observed and videotaped either during infant free or structured play. The videotapes were subsequently coded and analyzed either by trained researchers using a rating scale. Observations were either conducted in the home or in a lab some as early as 2 months, ${ }^{[42]} 8$ months postpartum, ${ }^{[13]}$ or at 2,4 , and 6 months. ${ }^{[44]}$

The length of the observations varied in addition to the period that the observations were coded. Boyd, Zayas, McKee ${ }^{[43]}$ observed mothers interact with her infant for a period of 20 minutes, then coding was completed by experts using the Interaction Rating Scale (IRS) for 3 minutes after fastforwarding tapes to 5 minutes. This scale examines separate categories for the mother and the infant. Some of the behaviors observed included facial expressions, gaze, and how the mother or infant expressed themselves vocally. ${ }^{[43]} \mathrm{Ob}$ servation times varied from 3 to 5 minutes as in the study by Stanley, Murray, Stein ${ }^{[42]}$ or 30 minutes. ${ }^{[48]}$ Once observations were made either questionnaires were used or scales to evaluate interactions.

To measure maternal-infant interaction, the majority of the studies utilized observations that were subsequently coded or a combination of observations and interviews with the mother. In Hererra, Reissland, Shepherd, ${ }^{[37]}$ interactions were coded and measured using the Observer System. Flykt, Kanninen, Sinkkonen, Punamäki ${ }^{[36]}$ used the Care Index and Boyd, Zayas, McKee ${ }^{[43]}$ measured interactions using the Interaction Rating Scale (IRS). Murray, Fiori-Cowley, Hooper, Cooper ${ }^{[41]}$ further observed interactions; however, specific measures were not indicated. Similarly Campbell, Cohn, Meyers ${ }^{[44]}$ coded observations of interactions; however, maternal-infant interactions were measured using the National Institute of Child Health and Human Development (NICHD). In this study, observations were again videotaped, however during structured play versus free play. Interactions were then coded by a scale that was developed by the NICHD, Qualitative Ratings for Parent-Child Interaction, which examined mother's behaviors such as sensitivity or intrusiveness. The qualitative study by Hoffman, Drotar ${ }^{[47]}$ measured interactions using the Global Dimensions Rating Scale (GDRS) to measure "Expressivity/Affective Involvement", "Stimulation/Activity", and "Responsivity/Sensitivity". This scale was specifically developed for this particular study. The other measure used was a version of the Greenspan-Lieberman Observation Scale to determine the frequency of behaviors. Face to face interactions, as in the study by Stanley, Murray, Stein $^{[42]}$ were videotaped and then observed for mothers' 
likelihood to respond to infant's behavior during a time period. Observations were further made during periods where mother's behaviors were noted that were not related to the infant's behavior. Observed interactions between the mother and child and like similar studies assessed and coded for how mother and infant connected. Maternal responsiveness was another ICC related concept that was studied in Forman, O'Hara, Stuart, Gorman, Larsen. ${ }^{[45]}$ In this study responsiveness was measured with the global ratings and a microscopic system to code for the interactions. Global ratings assessed maternal sensitivity, acceptance, and cooperation for each interaction between mother and child. ${ }^{[45]}$

Questionnaires and interviews were used to measure perceived ICC were further used. The Parent-Child Dysfunctional Interaction (P-CDI) subscale of the Parenting-Stress Index-Short Form (PSI-SF) assessed the mother's frustration with the interactions. ${ }^{[49]}$ In addition the Maternal Postnatal Attachment Scale (MPAS) measured how attached a mother felt towards their infant. ${ }^{[49]}$ Interviews were conducted in two studies. The Pregnancy and Childbirth Interview, Marital Relationship Interview, and the Motherhood Interview examined the feelings mothers had towards pregnancy, their relationship and motherhood respectively. ${ }^{[34]}$

To measure maternal-infant bonding, the Postpartum Bonding Questionnaire (PBQ) was used by Moehler, Brunner, Wiebel, Reck, Resch ${ }^{[40]}$ and Sockol, Epperson, Barber. ${ }^{[50]}$ Bonding was assessed using a 25-item questionnaire that includes four areas: "Impaired Bonding", "Rejection/Pathological Anger", "Maternal Anxiety", and "Risk of Abuse". In one study both the IRS and the PBQ were utilized. ${ }^{[39]}$ Maternal sensitivity was measured using two different scales. In the study by Kemppinen, Kumpulainen, Moilanen, Ebeling, ${ }^{[38]}$ the CARE-Index ${ }^{[54]}$ was used after observing interactions between the mother and the infant and coding captured maternal and infant vocal and facial expressions. Other measures for sensitivity were not specified; however mothers were observed and filmed for 10 minutes using toys that were provided for them. Researchers observed and coded for interactions that included how a mother responds to her infant's cries. ${ }^{[13]}$ In the study conducted by Barr, ${ }^{[33]}$ interviews and journals were completed by mothers to determine maternal adaptation or how they felt about becoming a mother. In Foreman, Henshaw, ${ }^{[51]}$ objective and subjective feelings of how a mother felt towards her infant and the role of becoming a mother were studied.

Maternal role attainment was examined using three different scales, "The Myself As Mother scale", "My Baby", and the "Perceived Competence Scale".[46] Maternal self-efficacy and emotional availability were measured using the Per-

Published by Sciedu Press ceived Maternal Parental Self-Efficacy scale ${ }^{[20]}$ and the Emotional Availability Scales (EAS) ${ }^{[48]}$ respectively.

\subsection{Variables explaining ICC in the context of PPD: Quantitative studies}

While results varied between studies, variables that explained ICC among the depressed samples of mothers included: demographics, severity of depression symptoms, timing of the depressive symptoms, social support, maternal adversity, attachment, and infant characteristics such as age, gender, or infant behaviors.

Demographics. Mason, Briggs, Silver ${ }^{[49]}$ found that having a higher level of education influenced ICC, or specifically, decreased feelings of stress in the mother's interactions. Although there was an association between education and interaction quality, the relationship was weak. Contrary to those findings, Moehler, Brunner, Wiebel, Reck, Resch ${ }^{[40]}$ examined the relationship between education level and bonding, however no association between the two was found.

Depression symptoms, severity, chronicity, and timing. Women with depressive symptoms were observed to have reduced ICC. Specifically, depressed women have more negative perceptions of their infants than perceptions of either themselves as a mother or their ability to care for their infants. ${ }^{[46]}$ Further, women with depression had more negative perceptions of their infants than never depressed women. ${ }^{[51]}$ In contrast, Kim, Teti, Cole ${ }^{[48]}$ found that emotional availability did not relate to maternal depressive symptoms.

With respect to depression severity, Loh, Vostanis ${ }^{[39]}$ did not find an association between depression severity and bonding. However, Sockol, Epperson, Barber ${ }^{[50]}$ found that higher scores on the EPDS (>11) or mothers reporting a symptom of severe depression predicted lower scores on the PBQ. The timing of depression symptoms is another explanatory variable of ICC. Flykt et al. ${ }^{[36]}$ found that prenatal depressive symptoms demonstrated a larger impact on the maternalinfant interaction than postnatal symptoms. In this study, mothers were less responsive with their children when depressive symptoms occurred prenatally. Similarly, mothers with chronic depression versus those whose symptoms diminish over time were less positive during interactions. ${ }^{[44]}$ Kemppinen et al. ${ }^{[38]}$ also found that chronic and prenatal depressive symptoms predicted negative maternal-infant interactions. Stanley, Murray, Stein ${ }^{[42]}$ did not find that chronic depressive symptoms impacted interactions. When responsiveness towards the infant and maternal depression were measured along 3 and 6 months periods, no significant relationship was found.

Social support. Only one study identified social support 
as an explanatory variable. Shorey, Chan, Chong, $\mathrm{He}^{[20]}$ revealed that social support, specifically, total support was a predictor of ICC, specifically, maternal parental self-efficacy (MPSE). According to Shorey, Chan, Chong, $\mathrm{He}^{[20]}$ higher levels of support, both functional and structural, were related to higher levels of MPSE.

Personal adversities. Social or personal adversities were further identified as variables that impacted ICC among women with depression. Loh, Vostanis ${ }^{[39]}$ observed that decreased satisfaction with work based on responses on the Social Questionnaire was correlated with poor maternal-infant interactions. Murray et al. ${ }^{[41]}$ measured personal and social adversities with the Life Events and Difficulties Schedule (LEDS). Some of the areas covered were infant health, relationships with their partners, or family health. The presence of adversities in mothers was associated with less sensitive interactions, decreased affirmations towards her infant and an increase in negating behaviors, such as disrupting infant activities. ${ }^{[41]}$ In Boyd, Zayas, McKee,${ }^{[43]}$ the Revised Life Events Questionnaire (LEQ) was used to study adversities. A few examples of life events included difficulties at work, acquiring new friendships, or relocating to a new city. The total number of negative life events determined the negative life events score. Although the two were correlated, the significance was small and life events predicted lower quality maternal-infant interactions.

Fetal and adult attachment. Maternal-fetal attachment, measured using the Maternal-Fetal Attachment scale was utilized in the study conducted by Alvarenga, Dazzani, Lordelo, Alfaya, Piccinini. ${ }^{[13]}$ The study examined a mother's attachment to her fetus and the relationship to maternal sensitivity. Researchers discovered that a strong maternal-fetal attachment during pregnancy was significantly correlated to ICC, specifically, greater maternal sensitivity at 8 months postpartum. Flykt, Kanninen, Sinkkonen, Pumamäki ${ }^{[36]}$ also studied prenatal attachment. Mothers who were more autonomously attached to their significant other were more sensitive in their interactions with their infants.

Infant characteristics. Infant characteristics, such as age, gender or infant behaviors were observed to impact ICC among depressed women. Azak, Raeder ${ }^{[35]}$ measured infant negative reactivity (a measure of temperament) and infant cognitive development. Researchers found that infant negative reactivity was negatively related to maternal style and positive cognitive development was related to dyadic mutuality. Similarly, Hoffman, Drotar ${ }^{[47]}$ found that infants of depressed mothers were observed to have a decreased range of emotions and affect during maternal-infant interactions. The infants also demonstrated a decrease in contingent be- haviors during interactions.

Infant age and gender were two other variables that were studied that may influence ICC among depressed mothers. Herrera, Reissland, Shepherd ${ }^{[37]}$ noted that depressed mothers differed in the patterns of lifting based on the infant's age. Ten-month-old infants of depressed mothers were lifted more frequently than 6-month-old infants of depressed mothers. Depressed mothers lifted their older infants more frequently as a form of restricting or controlling their behaviors. Infant gender was also researched in studies conducted by Murrary, Fiori-Cowley, Hooper, Cooper ${ }^{[41]}$ and Moehler, Brunner, Wiebel, Reck, Resch. ${ }^{[40]}$ Neither study revealed any effects of infant gender on ICC, specifically maternal-infant interactions and postpartum bonding. ${ }^{[40]}$

\subsection{Variables explaining ICC in the context of PPD: Qualitative studies}

One variable noted to impact ICC among depressed mothers. In Barr, ${ }^{[33]}$ the perception of being "stuck" in what is described as a liminal state influenced the interactions the mother had with her infant. The feeling of being stuck in this state decreased parenting abilities. For example, mothers described infant care tasks as being mechanical; having limited maternal-infant interactions when performing infant care tasks.

Infant behaviors were another explanatory variable of ICC, similar to what was observed among the qualitative studies. Frizzo, Vivian, Piccinini, Lopez ${ }^{[34]}$ determined that the infant's role in communication would impact the mother's interactions. In other words, if her infant was able to communicate with the mother and understand the mother, the mother was able to interact with her infant and pick up on infant cues.

\section{Discussion}

An integrative systematic review was completed to answer the question: "How do depression and other variables influence and/or explain ICC in the context of PPD?" While a significant amount of research has been conducted on how PPD affects maternal-infant interaction or performed ICC, little is known explicitly about variables that affect perceived maternal ICC. The systematic search showed that many of the included studies assessed/observed maternal-infant interaction (performed ICC) and eight measured perceptions of mothering or ICC with self-report scales or qualitative interviews. Among the included studies, eight common explanatory variables were found. The variables included: demographic variables such as education, severity of depressive symptoms, timing of depressive symptoms, social support, maternal adversity, attachment pattern (adult and fetal), ma- 
ternal characteristics such as perceptions, as well as infant reactivity or infant behaviors.

Demographic variables, particularly the level of education, predicted ICC. Depressed mothers with higher education levels reported lower stress in the maternal-infant interaction. ${ }^{[49]}$ A mother's education level appeared to be a protective factor in the maternal-infant relationship. Similar findings were observed in other reviews that examined parenting competence and maternal bonding. Johnson ${ }^{[55]}$ found the mother's education level, age, and socioeconomic status were factors that improved maternal-infant interaction. Similar findings were found in studies of non-depressed mothers, in particular a positive correlation was found between increased age, education level, and improved maternal competence or selfefficacy. ${ }^{[19]}$ In contrast, Moehler, Brunner, Wiebel, Reck, $\operatorname{Resch}^{[40]}$ did not find any significant relationship between education and bonding. The differences may be related to how ICC was measured. Mason, Briggs, Silver ${ }^{[49]}$ measured parenting stress, while Moehler, Brunner, Wiebel, Rec, $\operatorname{Resch}^{[40]}$ measured postpartum bonding, which may explain differences in research findings. For depressed mothers, higher education levels may act as a buffer to ICC. Higher levels of education may provide mothers with the skills to access resources and work effectively through parenting challenges that is similar to findings on non-depressed mothers.

Two studies showed that depressive symptoms predict negative perceptions of caregiving, potentially further decreasing feelings of competency and ICC in women. ${ }^{[46,51]}$ The timing of depressive symptoms and chronicity of symptoms were further found to be explanatory variables of ICC. Among the sample of mothers already diagnosed with depression, the timing of symptoms impacted ICC, specifically in observed maternal-infant interactions. Higher scores on the EPDS $(>11)^{[56]}$ or depressive symptoms occurring pre and postnatally negatively affected maternal style. ${ }^{[38]}$ Sockol, Epperson, Barber ${ }^{[56]}$ suggest that the severity of depressive symptoms may be an important explanatory variable of ICC, specifically bonding between a mother and infant. As well, mothers with chronic symptoms that remained over a 6-month period demonstrated decreased ICC, ${ }^{[44]}$ specifically observed to be less competent during activities such as feeding and to demonstrate more negative affect during toy play. ${ }^{[44]}$ In addition, mothers with symptoms diagnosed prenatally and at 5 months responded less to infants during interactions. ${ }^{[36]}$ Few researchers have studied how chronicity or higher depression scores influences ICC. Sockol et al.' ${ }^{\left[{ }^{[0]}\right.}$ finding of severity of symptoms demonstrating a weak relationship with maternal-interactions should therefore be interpreted with caution.
Social support was another variable that explained ICC among mothers with depression. Shorey, Chan, Chong, $\mathrm{He}^{[57]}$ showed a positive relationship between total social support and MPSE, with higher total social support scores positively correlated with higher scores of self-efficacy, in spite of depressive symptoms. Social support in the form of informational, instrumental or informal support helps improve mother's feelings of adequacy in performing tasks related to infant care. ${ }^{[57]}$ These findings are consistent with other studies that examined the positive role that social support plays in mothers with or without depression. ${ }^{[6,19]}$ Findings are also consistent with Barnard's model ${ }^{[58-60]}$ where the environment, which includes social support, is associated with improved ICC.

Maternal adversities or stressful events for mothers affected by depression were further identified as explanatory variables of ICC. For depressed mothers, recent adversities, such as a difficult relationship or decrease in job satisfaction, lowered maternal-infant interaction quality. A decrease in interaction quality suggests that adversities and depression are related. ${ }^{[61]}$ According to Najman et al. ${ }^{[61]}$ adversities may worsen depressive symptoms further impacting the way the mother functions, therefore decreasing parenting abilities. Adversities have been shown to lead to distress, negatively impact mood, and decease a mother's own self-perception. ${ }^{[62]}$ Poor self-perception in conjunction with depressive symptoms may further disrupt ICC. Other research has shown that earlier life adversities, such as those in childhood, predict both depressive symptoms and less than optimal child developmental outcomes. This suggests that future research could examine the associations among early life adversities, maternal depression and ICC. ${ }^{[63]}$

A mother's sense of attachment to her fetus has been correlated with improved ICC. Research conducted by Alvarenga, Dazzani, Lordelo, Alfaya, Piccinini ${ }^{[13]}$ noted that better maternal-fetal attachment scores were positively correlated with stronger sensitivity scores. ${ }^{[36]}$ Maternal sensitivity examines how a mother is able to pick up on infant cues or signals. ${ }^{[64]}$ Mothers with depression often have difficulty with these cues due to symptoms related to low mood that prevent mothers from being attuned to the needs of the infant. ${ }^{[65]}$ However, as Alvarenga, Dazzani, Lordelo, Alfaya, Piccinini ${ }^{[13]}$ demonstrated that, mothers with strong fetal attachment demonstrate increases in sensitivity. While similar findings have been observed with non-depressed women, ${ }^{[64]}$ results again should be taken with caution due to the study's low quality score and small sample size.

Infant characteristics were identified as explanatory variables of ICC in depressed mothers, including infant temperament 
and age. These findings are consistent with similar research done on non-depressed samples. Research has shown that mothers of infants with easier temperament have less difficulty with parenting abilities. ${ }^{[6,19]}$ Responsive infants create positive maternal feeling states and confidence carrying out infant care tasks. In one study, data revealed that infant age influenced ICC, in that ten-month-old infants were lifted and held more than 6-month-old infants, ${ }^{[37]}$ with the authors suggesting that depressed mothers had difficulty attending to infant cues as the infant ages. These findings are consistent with Barnard's model, ${ }^{[58-60]}$ where newborns' developmental age can influence competency. Infant gender was studied in two separate studies, however no significant associations were obtained on infant gender and competence. ${ }^{[40,41]}$

To our knowledge, this is the first narrative systematic review that examined explanatory variables of ICC among women with depression. The review was comprehensive in focusing on up to 24 months postpartum and no imposed restrictions on the years of the studies reviewed, adding to the strength of the study. One limitation is that many of the variables identified were from studies that used self-report measures of depression and perceived ICC. There is the possibility of bias in the reporting of responses i.e., over reporting symptoms that could influence the results of this review. Another limitation is the number of qualitative studies, with only two. Future research is needed on qualitative investigation of perceived ICC and depression in order to obtain a broad spectrum of information and perspectives. Furthermore, while questions about the quality of articles were assessed with expert advice, it would have been helpful to consult a second ICC expert. Finally, fathers were not considered for this systematic review due to a lack of research that has been conducted on explanatory variables of ICC among fathers with postpartum depression.

Given the link between PPD and child development may be mediated by the quality of maternal-infant relationship, ${ }^{[66]}$ knowledge of explanatory variables of ICC can inform early interventions and treatment. Early treatment can prevent the negative long-term impact on children's development associated with altered relationships between mothers with PPD and their infants. Early treatment such as counseling could also increase maternal self-confidence, and put less strain on the health care system in the future associated with remedial support for children with developmental problems. ${ }^{[15]}$ Standard questions could be included in all assessments for mothers who have been diagnosed with PPD in order to assess other predictors of ICC.

Further research on ICC should also include fathers. Many studies have a focus on the mothers' ability in providing care. Given fathers' seminal role in support provision to mothers with $\mathrm{PPD},{ }^{[67]}$ examining the impact of a father's perception in ICC and its impact on infants may require additional exploration. Finally, research on the role of cultural influences on ICC may add to the research findings. The majority of the research was conducted predominantly on Caucasian mothers. Few of the studies researched mothers of different races and did not fully explore how a mother's race may play a role in ICC and how it may influence mothers and their families in obtaining support. Finally, the lack of findings on perceived ICC demonstrates the need for further research on this topic area. Policy makers who make decisions about funding programs that serve perinatal women should focus on the development of social support groups for women who lack support systems and who are at risk for PPD. In addition, developing practice guidelines is imperative for nurses to specifically focus on mothers who describe having adversities in their life. Proper identification could act as a screening tool for possible counseling.

\section{Conclusion}

In summary, to improve ICC among women with depression, understanding the variables that may influence perceived and performed competency is essential to help parents achieve their developmental potentials. Variables that predicted ICC included: sociodemographic variables such as education, severity of depressive symptoms, timing of depressive symptoms, social support, maternal adversity, attachment pattern (adult and fetal), as well as infant temperamental reactivity. This knowledge may lead to early detection of areas for focus in helping mothers attain optimal ICC and ultimately prevent possible developmental problems in their children. For women with PPD, assessments of sociodemographics, symptoms and timing of depression, social support and attachment may lead to development of targeted programs to promote ICC.

\section{ACKNOWLEDGements}

We would like to thank Dr. Loretta Secco for her valuable guidance with regard to determining similar concepts to ICC with different names.

\section{REFERENCES}

[1] Lanes A, Kuk JL, Tamim H. Prevalence and characteristics of postpartum depression symptomatology among Canadian women: A cross-sectional study. BMC Public Health. 2011; 11(1): 302.
PMid:21569372 https: //doi .org/10.1186/1471-2458-11-3 02

[2] Pearlstein T, Howard M, Salisbury A, et al. Postpartum depression. 
Am J Obstet Gynecol. 2009; 200(4): 357-364. PMid:19318144 https://doi.org/10.1016/j.ajog.2008.11.033

[3] American Psychological Association. Diagnostic and statistical manual of mental disorders. American Psychiatric Association; 2013.

[4] Leger J, Letourneau N. New mothers and postpartum depression: a narrative review of peer support intervention studies. Health Soc Care Comm. 2015; 23(4): 337-348. PMid:25346377 https: //doi.org/10.1111/hsc.12125

[5] Secco L. The infant care questionnaire: Assessment of reliability and validity in a sample of healthy mothers. J Nurs Meas. 2002; 10(2): 97-110. https://doi.org/10.1891/jnum.10.2.97.52555

[6] Ngai FW, Chan SWC, Ip WY. Predictors and correlates of maternal role competence and satisfaction. Nurs Res. 2010; 59(3): 185-193. PMid:20404775 https://doi.org/10.1097/NNR.0b013e3181 dbb9ee

[7] Mercer RT. Becoming a mother versus maternal role attainment. J Nurs Scholarship. 2004; 36(3): 226-232. https ://doi .org/10.1 $111 / j .1547-5069.2004 .04042 . x$

[8] andura A. Self-efficacy: The exercise of control. Macmillan; 1997.

[9] Goldberg S. Social competence in infancy: A model of parent-infant interaction. Merrill-Palmer Q Beh. 1977; 23(3): 163-177.

[10] Barnard K, Hammond M, Booth C, et al. Measurement and meaning of parent-child interaction. In: F Morrison, C Lord, D Keating, eds. Applied Developmental Psychology. New York City, NY: Academic; 1989.

[11] Field T. Postpartum depression effects on early interactions, parenting, and safety practices: a review. Infant Behav Dev. 2010; 33(1): 16. PMid:19962196 https://doi.org/10.1016/j.infbeh. 200 9.10.005

[12] Booth CL, Barnard KE, Mitchell SK, et al. Successful intervention with multi-problem mothers: Effects on the motherinfant relationship. Inft Ment Health J. 1987; 8(3): 288306. https://doi .org/10.1002/1097-0355(198723) 8:3<28 8: : AID-IMHJ2280080311>3.0.C0;2-Q

[13] Alvarenga P, Dazzani MVM, Lordelo EdR, et al. Predictors of sensitivity in mothers of 8-month-old infants. Paidéia (Ribeirão Preto). 2013; 23(56): 311-320. https://doi.org/10.1590/1982-432 72356201305

[14] Letourneau N, Secco L, Colpitts J, et al. Quasi-experimental evaluation of a telephone-based peer support intervention for maternal depression. J Adv Nurs. 2015; 71(7): 1587-1599. PMid:25705786 https://doi.org/10.1111/jan.12622

[15] Poobalan AS, Aucott LS, Ross L, et al. Effects of treating postnatal depression on mother-infant interaction and child development: systematic review. Brit J Psychiat. 2007; 191(5): 378-386. PMid:17978316 https://doi.org/10.1192/bjp.bp.106.032789

[16] Secco ML, Ateah C, Woodgate R, et al. Perceived and performed infant care competence of younger and older adolescent mothers. Issues Comp Pediatr Nurs. 2002; 25(2): 97-112. PMid:12060517 https://doi.org/10.1080/01460860290042521

[17] Beck CT. The effects of postpartum depression on child development: a meta-analysis. Arch Psychiat Nurs. 1998; 12(1): 12-20. https ://doi.org/10.1016/S0883-9417 (98) 80004-6

[18] O'Connor E, Rossom RC, Henninger M, et al. Primary care screening for and treatment of depression in pregnant and postpartum women: evidence report and systematic review for the US Preventive Services Task Force. Jama. 2016; 315(4): 388-406. PMid:26813212 https://doi.org/10.1001/jama.2015.18948

[19] Bryanton J, Gagnon AJ, Hatem M, et al. Predictors of early parenting self-efficacy: results of a prospective cohort study. Nurs Res. 2008; 57(4): 252-259. PMid:18641494 https ://doi .org/10.1097/01 .NNR.0000313490.56788.cd

Published by Sciedu Press
[20] Shorey S, Chan SWC, Chong YS, et al. Predictors of maternal parental self-efficacy among primiparas in the early postnatal period. Western J Nurs Res. 2015; 37(12): 1604-1622. PMid:24906360 https://doi.org/10.1177/0193945914537724

[21] Robertson E, Grace S, Wallington T, et al. Antenatal risk factors for postpartum depression: a synthesis of recent literature. Gen Hosp Psychiat. 2004; 26(4): 289-295. PMid:15234824 https: //doi.org/10.1016/j.genhosppsych.2004.02.006

[22] Dennis CLE. Treatment of postpartum depression, part 2: a critical review of nonbiological interventions. J Clin Psychiat. 2004; 65(9): 1252-1265. https://doi . org/10.4088/JCP.v65n0915

[23] Doucet S, Letourneau N, Blackmore ER. Support needs of mothers who experience postpartum psychosis and their partners. J Obst Gyn Neo. 2012; 41(2): 236-245. PMid:22375839 https: //doi.org/10.1111/j.1552-6909.2011.01329.x

[24] Sit D, Rothschild AJ, Wisner KL. A review of postpartum psychosis. J Womens Health. 2006; 15(4): 352-368. PMid:16724884 https://doi.org/10.1089/jwh.2006.15.352

[25] Hipwell A, Goossens F, Melhuish E, et al. Severe maternal psychopathology and infant-mother attachment. Dev Psychopathol. 2000; 12(2): 157-175. PMid:10847622 https://doi.org/10.1 017/S0954579400002030

[26] Lawrie T, Herxheimer A, Dalton K. Oestrogens and progestogens for preventing and treating postnatal depression. Cochrane Database Syt Rev. 2000.

[27] Korja R, Savonlahti E, Ahlqvist-Björkroth S, et al. Maternal depression is associated with mother-infant interaction in preterm infants. Acta Paediatrica. 2008; 97(6): 724-730. PMid:18373715 https://doi.org/10.1111/j.1651-2227.2008.00733.x

[28] Milliones J. Relationship between perceived child temperament and maternal behaviors. Child Dev. 1978; 1255-1257.

[29] Whittemore R, Knafl KJJoan. The integrative review: Updated methodology. J Adv Nurs. 2005; 52(5): 546-553. PMid:16268861 https://doi.org/10.1111/j.1365-2648.2005.03621.x

[30] Field T. Prenatal depression effects on early development: A review. Infant Behav Dev. 2011; 34(1): 1-14. PMid:20970195 https: //doi.org/10.1016/j.infbeh.2010.09.008

[31] Mäntymaa M, Puura K, Luoma I, et al. Mother's early perception of her infant's difficult temperament, parenting stress and early mother-infant interaction. Nord J Psychiatr. 2006; 60(5): 379-386. PMid:17050296 https://doi.org/10.1080/08039480600937 280

[32] Young JM, Solomon MJ. How to critically appraise an article. Nat Rev Gastroenterol Hepatol. 2009; 6(2): 82. PMid:19153565 https://doi.org/10.1038/ncpgasthep1331

[33] Barr JA. Postpartum depression, delayed maternal adaptation, and mechanical infant caring: A phenomenological hermeneutic study. Int J Nurs Stud. 2008; 45(3): 362-369. PMid:17157848 https: //doi.org/10.1016/j.ijnurstu.2006.10.002

[34] Frizzo GB, Vivian AG, Piccinini CA, et al. Crying as a Form of Parent-Infant Communication in the Context of Maternal Depression. J Child Fam Stud. 2013; 22(4): 569-581. https ://doi .org/10.1 007/s10826-012-9612-2

[35] Azak S, Raeder S. Trajectories of parenting behavior and maternal depression. Infant Behav Dev. 2013; 36(3): 391-402. PMid:23603820 https://doi.org/10.1016/j.infbeh.2013.03.004

[36] Flykt M, Kanninen K, Sinkkonen J, et al. Maternal depression and dyadic interaction: the role of maternal attachment style. Infant Chil Dev. 2010; 19(5): 530-550.

[37] Herrera E, Reissland N, Shepherd J. Maternal touch and maternal child-directed speech: effects of depressed mood in the postnatal 
period. J Affect Disorders. 2004; 81(1): 29-39. PMid:15183597 https://doi.org/10.1016/j.jad.2003.07.001

[38] Kemppinen K, Kumpulainen K, Moilanen I, et al. Recurrent and transient depressive symptoms around delivery and maternal sensitivity. Nord J Psychiat. 2006; 60(3): 191-199. PMid:16720509 https://doi.org/10.1080/08039480600635975

[39] Loh CC, Vostanis P. Perceived mother-infant relationship difficulties in postnatal depression. Infant Child Dev. 2004; 13(2): 159-171. https://doi.org/10.1002/icd.347

[40] Moehler E, Brunner R, Wiebel A, et al. Maternal depressive symptoms in the postnatal period are associated with long-term impairment of mother-child bonding. Arch Women Ment Hlth. 2006; 9(5): 273 278. PMid:16937313 https ://doi.org/10.1007/s00737-006 $-0149-5$

[41] Murray L, Fiori-Cowley A, Hooper R, et al. The impact of postnatal depression and associated adversity on early mother-infant interactions and later infant outcome. Child Dev. 1996; 67(5): 2512-2526. https://doi.org/10.2307/1131637

[42] Stanley C, Murray L, Stein A. The effect of postnatal depression on mother-infant interaction, infant response to the still-face perturbation, and performance on an instrumental learning task. Dev Psychopathol. 2004; 16(1): 1-18. PMid:15115062

[43] Boyd RC, Zayas LH, McKee MD. Mother-infant interaction, life events and prenatal and postpartum depressive symptoms among urban minority women in primary care. Matern Child Healt J. 2006; 10(2): 139. PMid:16397831 https://doi.org/10.1007/s10995 $-005-0042-2$

[44] Campbell SB, Cohn JF, Meyers T. Depression in first-time mothers: mother-infant interaction and depression chronicity. Dev Psychol. 1995; 31(3): 349. https://doi.org/10.1037/0012-1649.31 .3 .349

[45] Forman DR, O'Hara MW, Stuart S, et al. Effective treatment for postpartum depression is not sufficient to improve the developing mother-child relationship. Dev Psychopathol. 2007; 19(2): 585-602.

[46] Fowles ER. The relationship between maternal role attainment and postpartum depression. Health Care Women In. 1998; 19(1): 83-94. PMid:9479097 https://doi.org/10.1080/073993398246601

[47] Hoffman Y, Drotar D. The impact of postpartum depressed mood on mother-infant interaction: Like mother like baby? Inft Ment Health J. 1991; 12(1): 65-80. https://doi.org/10.1002/1097-0355(19 9121) $12: 1<65:$ : AID-IMHJ2280120107>3.0.CO;2-T

[48] Kim BR, Teti DM, Cole PM. Mothers' affect dysregulation, depressive symptoms, and emotional availability during mother-infant interaction. Inft Ment Health J. 2012; 33(5): 469-476. PMid:28520269 https://doi.org/10.1002/imhj.21326

[49] Mason Z, Briggs R, Silver E. Maternal attachment feelings mediate between maternal reports of depression, infant social-emotional development, and parenting stress. J Reprod Infant Psyc. 2011; 29(4): 382-394. https://doi.org/10.1080/02646838.2011.629994

[50] Sockol LE, Battle CL, Howard M, et al. Correlates of impaired mother-infant bonding in a partial hospital program for perinatal women. Arch Women Ment Hlth. 2014; 17(5): 465-469. PMid:24643421 https://doi .org/10.1007/s00737-014-041 9-6

[51] Foreman DM, Henshaw C. Objectivity and subjectivity in postnatally depressed mothers' perceptions of their infants. Child Psychiat Hum D. 2002; 32(4): 263-275. https://doi.org/10.1023/A: 1015266410308
[52] Zimmerman M, Coryell W. The inventory to diagnose depression, lifetime version. Acta Psychiatr. 1987; 75(5): 495-499. https: //doi.org/10.1111/j.1600-0447.1987.tb02824.x

[53] Aben I, Verhey F, Lousberg R, et al. Validity of the beck depression inventory, hospital anxiety and depression scale, SCL-90, and hamilton depression rating scale as screening instruments for depression in stroke patients. Psychosomatics. 2002; 43(5): 386393. PMid:12297607 https://doi.org/10.1176/appi.psy.4 3.5.386

[54] Crittenden P, Kozlowska K, Landini A. Assessing attachment in school-age children. Clin Child Psychol P. 2010; 15(2): 185-208. PMid:20176770 https://doi.org/10.1177/13591045093567 41

[55] Johnson K. Maternal-Infant Bonding: A Review of Literature. Int J Childbirth Educ. 2013; 28(3).

[56] Sockol LE, Epperson CN, Barber JP. The relationship between maternal attitudes and symptoms of depression and anxiety among pregnant and postpartum first-time mothers. Arch Women Ment Hlth. 2014; 17(3): 199-212. PMid:24643422 https://doi.org/10.1 007/s00737-014-0424-9

[57] Shorey S, Chan SWC, Chong YS, et al. Maternal parental selfefficacy in newborn care and social support needs in Singapore: a correlational study. J Clin Nurs. 2014; 23(15-16): 2272-2283.

[58] Barnard K, Eyres S, Lobo M, et al. In: Brazelton TB, Lester BM, eds. An ecological paradigm for assessment and intervention. New York, NY: Elsevier Science Publishing Co; 1983; 200-218.

[59] Barnard KE, Hammond M, Mitchell SK, et al. Caring for high-risk infants and their families. In: R Starr, ed. The psychosocial aspects of the family. Lexington, MA: Lexington Books; 1985; 245-266.

[60] Snyder C, Eyres SJ, Barnard K. New findings about mothers' antenatal expectations and their relationship to infant development. MCN Am J Matern Child Nurs. 1979; 4(6): 354-357. PMid:114729

[61] Najman JM, Plotnikova M, Williams G, et al. Maternal depression and family adversity: Linked pathways to offspring depression? J Psychiat Res. 2017; 88: 97-104.

[62] Keinan G, Shrira A, Shmotkin D. The association between cumulative adversity and mental health: considering dose and primary focus of adversity. Qual Life Res. 2012; 21(7): 1149-1158. PMid:21983715 https://doi.org/10.1007/s11136-011-0035-0

[63] McDonnell CG, Valentino K. Intergenerational effects of childhood trauma: evaluating pathways among maternal ACEs, perinatal depressive symptoms, and infant outcomes. Child Maltreatment. 2016; 21(4): 317-326. PMid:27457410 https : //doi .org/10.1177/10 77559516659556

[64] Shin H, Park YJ, Kim MJ. Predictors of maternal sensitivity during the early postpartum period. J Adv Nurs. 2006;55(4):425-434 PMid:16866838 https://doi.org/10.1111/j.1365-2648.20 06.03943.x

[65] Letourneau NL, Tramonte L, Willms JS. Maternal depression, family functioning and children's longitudinal development. J Pediatr Nurs. 2013; 28(3): 223-234. PMid:22940454 https://doi.org/10.1 016/j.pedn . 2012.07.014

[66] Beck CT. Postpartum depressed mothers' experiences interacting with their children. Nurs Res. 1996; 45(2): 98-104.

[67] Letourneau NL, Dennis CL, Benzies K, et al. Postpartum depression is a family affair: addressing the impact on mothers, fathers, and children. Issues Ment Health N. 2012; 33(7): 445-457. PMid:22757597 https://doi.org/10.3109/01612840.2012.673054 